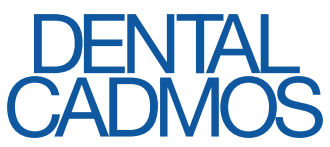

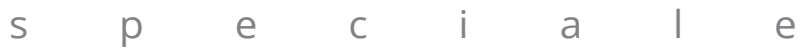 PARODONTOLOGIA
}

Passato, presente e futuro dei coadiuvanti locali e sistemici nel trattamento non chirurgico di perimplantiti e parodontiti: indicazioni e limiti

M. Mensi, E. Scotti, L. Francetti, M. Giargia, M. Latronico, A. Camurati, M.E. Guarnelli, R. Rotundo 


\title{
Passato, presente e futuro dei coadiuvanti locali e sistemici nel trattamento non chirurgico di perimplantiti e parodontiti: indicazioni e limiti
}

\author{
M. Mensi ${ }^{a}$, E. Scotti ${ }^{a}$, L. Francetti ${ }^{b}$, M. Giargiac, M. Latronico ${ }^{c}$, A. Camurati ${ }^{d}$, M.E. Guarnellie, R. Rotundo ${ }^{f}$ \\ a Università degli Studi di Brescia, Dipartimento di Specialità Chirurgiche, Scienze Radiologiche e Sanità Pubblica, Clinica Odontoiatrica \\ b IRCCS Istituto Ortopedico Galeazzi, Centro Odontoiatrico Universitario, Milano \\ c Università degli Studi di Genova, Corso di Laurea Specialistica in Odontoiatria e Protesi Dentaria \\ d Libero professionista in $\bullet . . . . . .$. \\ e Università degli Studi di Ferrara, Centro Interdipartimentale di Ricerca per lo Studio delle Malattie Parodontali e Perimplantari \\ f Università degli Studi di Firenze, Corso di Laurea Specialistica in Odontoiatria e Protesi Dentaria e Corso di Laurea in Igiene Dentale
}

Dall'indagine Antibiotici topici nel trattamento delle parodontiti e perimplantiti: quali indicazioni condotta da Edra su un campione di 1.000 odontoiatri italiani, e in fase di pubblicazione, è emersa l'esistenza di un'idea confusa circa le indicazioni all'utilizzo degli antibiotici in terapia parodontale. La maggioranza della popolazione intervistata dichiara:

- di conoscere il rischio di resistenza batterica indotta dalla somministrazione su larga scala di antibiotici sistemici;

- di conoscere I'elevata percentuale di patogeni resistenti all'amoxicillina;

- ciò nonostante, di prescrivere almeno settimanalmente antibiotici sistemici in particolare amoxicillina + acido clavulanico per la terapia di ascessi parodontali e perimplantari;

- di avvertire I'esigenza di un antibiotico topico efficace;

- di non conoscere la differenza tra "medical device" e "farmaco";

- di osservare una prevalenza di perimplantite nel proprio studio fino al $10 \%$.

È importante rilevare che sia il Ministero della Salute sia I'AIFA raccomandano un'attenta e mirata prescrizione degli antibiotici sistemici, data l'emergenza delle resistenze batteriche di cui siamo il Paese principalmente responsabile.
Da questi presupposti è scaturita la necessità di confronto tra parodontologi esperti in materia per discutere le attuali evidenze scientifiche e cliniche in merito alle problematiche sopra elencate.

\section{INTRODUZIONE}

Il concetto che i batteri svolgano un ruolo prioritario nell'eziologia della malattia perimplantare e parodontale è ben documentato. Obiettivo primario della terapia parodontale è eradicare e/o ridurre la carica batterica parodontopatogena attraverso il debridment meccanico e manuale.

Diversi protocolli di terapia parodontale non chirurgica si sono susseguiti nel corso degli anni, a partire dalla strumentazione manuale di Scaling e Root Planning (SRP) a quadranti alla più attuale Full Mouth Instrumentation (FMI) in combinazione o meno con la terapia antibiotica sistemica (amoxicillina + metronidazolo).

La scelta del protocollo terapeutico più adatto muove da una corretta diagnosi che, riprendendo quanto riporta Armitage [1], deve fondarsi su un'attenta analisi di dati clinici, radiografici e anamnestici (classificazione del 1999). Questo permette di distinguere le diverse categorie di malattia parodontale:

- gengivite; 
- parodontite cronica;

- parodontite aggressiva localizzata;

- parodontite aggressiva generalizzata;

- periodontite come manifestazione di malattia sistemica;

- malattie parodontali ulcero-necrotiche;

- ascesso parodontale;

- lesioni combinate endo-parodontali;

- lesioni parodontali indotte o acquisite (traumatiche).

I successivi step fondamentali per la gestione del paziente parodontale prevedono una rivalutazione post-trattamento ad almeno 6 settimane di distanza, l'impostazione di un followup personalizzato e I'eventuale correzione chirurgica di tasche residue.

L'esigenza di un trattamento aggiuntivo deriva dai limiti della terapia non chirurgica:

- abilità dell'operatore;

- tasche profonde;

- difficoltà di accesso;

- anatomia complessa;

- efficacia ed efficienza degli strumenti;

- capacità di alcuni batteri di infiltrare i tessuti molli.

Diversi coadiuvanti sono stati proposti in letteratura per migliorare gli outcome clinici e microbiologici ottenibili con la sola terapia non chirurgica:

- clorexidina;

- fluoruro amminico e fluoruro stannoso;

- terapia fotodinamica;

- laser;

- disidratante chimico a base di solfuri;

- antibiotici topici.

Dall'evidenza scientifica, così come dalla discussione tra gli esperti, si evince che i coadiuvanti topici proposti e testati a oggi migliorano i risultati della terapia non chirurgica in maniera limitata e spesso mostrano minime differenze, significative solo statisticamente e non certo da un punto di vista clinico.

\section{GLI ANTIMICROBICI}

È assodato che l'associazione di misure per il controllo del biofilm sia sopragengivale sia subgengivale determina un viraggio della flora microbica orale verso specie non patogene con conseguente miglioramento dei parametri biometrici parodontali. Questi cambiamenti, oltre a essere attribuibili all'effetto della strumentazione parodontale, potrebbero essere dovuti, almeno in parte, al rigoroso controllo domiciliare del biofilm implementato dall'utilizzo di antimicrobici locali. L'uso di antimicrobici locali a base di fluoruro amminico/fluoruro stannoso ( $\mathrm{AmF} / \mathrm{SnF}_{2}$ ) associato alle pratiche quotidiane di igiene orale domiciliare risulta maggiormente efficace in termini di controllo del biofilm e riduzione dell'infiammazione gengivale rispetto alle sole procedure meccaniche [2]. I risultati di un recente studio [3] indicano come I'adozione di un protocollo di igiene orale rigoroso, attuato mediante l'impiego di antimicrobici a base di $\mathrm{AmF} / \mathrm{SnF}_{2^{\prime}}$ possa controllare la ricolonizzazione batterica subgengivale da parte dei batteri parodontopatogeni in pazienti con elevata suscettibilità alla malattia parodontale, determinando una riduzione della carica microbica totale del $70 \%$ e del complesso rosso del $75 \%$ a 12 settimane dal trattamento.

La necessità di ottenere una più drastica e duratura riduzione della carica batterica nel cavo orale e in particolare nelle nicchie ecologiche subgengivali, in modo da ridurre il rischio di una ricolonizzazione precoce dei solchi/tasche parodontali, ha imposto I'adozione di regimi terapeutici con un esteso impiego anche a livello professionale dell'antimicrobico locale, nella fattispecie la clorexidina. In alcuni studi si è evidenziato come I'associazione della clorexidina al debridement parodontale possa indurre benefici aggiuntivi in termini di shift del microbiota subgengivale e miglioramento dei parametri clinici [4]. In altri studi un approccio simile ha dimostrato limitati effetti clinici e microbiologici rispetto allo stesso regime senza I'utilizzo della clorexidina [5]. In accordo, è stato dimostrato recentemente come I'uso professionale della clorexidina associato alla strumentazione parodontale non abbia indotto vantaggi aggiuntivi in termini clinici e microbiologici rispetto alla sola strumentazione [6]. La mancanza dell'effetto potrebbe essere dovuta in parte alla concentrazione dell'antisettico utilizzato per l'irrigazione (0,02\%). D'altronde anche quando sono state valutate concentrazioni maggiori di antisettico i risultati ottenuti sono stati ancora una volta limitati se non assenti [5]. In conclusione, i risultati degli studi riportati in letteratura sembrano indicare che non vi sia un beneficio aggiuntivo clinico e microbiologico quando la clorexidina viene associata alla strumentazione parodontale.

Nonostante la strumentazione parodontale associata a regimi rigorosi di controllo del biofilm risulti efficace nel miglioramento dei parametri clinici e microbiologici, essa non è però in grado di consentire la completa eradicazione delle specie batteriche patogene dalle tasche parodontali se non in un'esigua percentuale di pazienti. 


\section{LA TERAPIA FOTODINAMICA}

La terapia fotodinamica o disinfezione attivata dalla luce si avvale del principio che determinate sostanze, dette "fotosensibilizzanti", in presenza di ossigeno e di una sorgente luminosa tarata su specifiche lunghezze d'onda coerente (laser) o non (lampade a led) abbiano la capacità di agire specificamente contro i microrganismi senza provocare alcun danno alle cellule ospiti.

La terapia fotodinamica è stata proposta nella terapia causale della malattia parodontale: le revisioni sistematiche di studi scientifici a oggi non hanno dimostrato un reale beneficio in sostituzione ai trattamenti convenzionali, mentre la combinazione sembra mostrare dati positivi specie nelle zone difficilmente accessibili [7].

L'attuale discordanza dei dati di letteratura è dovuta probabilmente a vari fattori tra cui le differenze nei fotosensibilizzanti, nelle apparecchiature e nelle lunghezze d'onda impiegati nei singoli studi.

Nel trattamento della perimplantite i dati sperimentali e clinici attualmente disponibili dimostrano che la terapia fotodinamica può ridurre i batteri sulle superfici implantari senza causare effetti collaterali [8].

Gli effetti clinici della terapia fotodinamica rispetto ai metodi convenzionali di decontaminazione delle superfici implantari non possono essere valutati in quanto mancano studi clinici controllati e randomizzati con risultati a medio e lungo termine.

Poiché $\mathrm{i}$ dati che emergono dalla letteratura al momento disponibile sono discordanti e non forniscono indicazioni precise sull'efficacia, ulteriori studi scientifici potranno fare chiarezza.

\section{IL LASER}

Si ricorda che parlare genericamente di laser ha un significato alquanto limitato, infatti "laser" è semplicemente un acronimo (Light Amplification by Stimulated Emission of Radiation) che indica I'emissione di un fascio di luce - o meglio di una radiazione elettromagnetica, infatti molti laser (medicali e non) utilizzano lunghezze d'onda abbondantemente fuori dello spettro visibile - coerente, monocromatica e collimata; inoltre esistono laser medicali con caratteristiche fisiche e potenzialità, quantomeno teoriche, di interazione con i tessuti del tutti difformi tra loro.

In particolare, in odontoiatria i laser studiati e in qualche modo disponibili sono [9]:
- $\mathrm{CO}_{2}$ (laser ad anidride carbonica): ha indicazioni chirurgiche ed è in grado di tagliare i tessuti molli tramite un processo di carbonizzazione mediato dall'assorbimento dell'energia nell'acqua presente negli stessi. $\mathrm{Ha}$ ottenuto I'approvazione per uso odontoiatrico nel 1976;

- Nd:YAG (Neodimio:YAG): I'energia in questo caso è principalmente dispersa più che assorbita dai tessuti molli, soprattutto se molto pigmentati (utilità nella gestione delle neoformazioni vascolari), e duri. Il controllo della profondità di penetrazione risulta assai difficile. Non ha ottenuto alcuna approvazione per uso odontoiatrico da parte della Food and Drug Administration;

- Er:YAG (Erbium:YAG): fu il primo laser approvato nel 1997 per applicazioni ablative di tessuti duri. Presenta una captazione 1.500 volte superiore al precedente da parte dei tessuti; I'acqua presente viene rapidamente portata a ebollizione e ciò causa microesplosioni;

- laser a diodi: ha un funzionamento e un pattern di assorbimento molto simili al Nd:YAG ma con minori surriscaldamenti nella compagine dei tessuti. Da usarsi a contatto dei tessuti, restituisce un feedback molto simile a un elettrocauterio.

Altri argomenti che nel corso degli anni hanno spinto la ricerca verso le applicazioni cliniche di questi dispositivi sono $\mathrm{i}$ presunti, ma non dimostrati, vantaggi in termini di: minor sanguinamento, minor dolore e migliore e più rapida guarigione, effetti battericidi diretti, batteriostatici, biostimolanti.

Analizzando nello specifico le più recenti revisioni sistematiche della letteratura in merito alla laserterapia in clinica parodontale, si identificano tre lavori molto autorevoli condotti negli ultimi dieci anni: uno commissionato dall'American Academy of Periodontology nel 2006 [10] e due comparsi sul Journal of Clinical Periodontology rispettivamente nel 2008 [11] e nel 2014 [12].

La revisione del 2008 [11] evidenzia che I'eterogeneità dei disegni sperimentali, delle apparecchiature testate e delle caratteristiche dei laser non consente di effettuare una metanalisi, pertanto gli autori si limitano a un'analisi narrativa dei risultati: I'applicazione del laser Er:YAG in terapia parodontale non chirurgica sembra avere il miglior potenziale tra le diverse lunghezze d'onda e sembra produrre un risultato simile alla terapia convenzionale (benché vi siano dubbi sulla potenza statistica degli studi considerati). Per le altre lunghezze $d^{\prime}$ onda non è presente alcuna prova scientifica. Inoltre, per quanto concerne la sicurezza, negli studi esaminati non sono 
emersi effetti avversi alle intensità energetiche considerate. La revisione del 2014 [12] mostra la sostanziale equivalenza della monoterapia con laser Er:YAG rispetto allo SRP nel breve termine, ma a 6 e 12 mesi i dati sono insufficienti, mentre come aggiunta allo SRP la differenza tra I'usare o no il laser è statisticamente non significativa oltreché clinicamente irrilevante. Gli autori fanno inoltre notare che solo due degli studi inclusi nella metanalisi, che rappresentano quanto di metodologicamente migliore sia disponibile in letteratura in materia, soddisfacevano appieno le raccomandazioni CONSORT riguardo al controllo degli errori sistematici (bias). Numerosi studi sono stati condotti con I'intento di verificare I'efficacia del laser in parodontologia, e in particolare in terapia causale parodontale. Gran parte di questi tuttavia presenta limiti metodologici e statistici più o meno importanti che, di fatto, hanno suscitato dubbi sui risultati ottenuti. Uno studio clinico randomizzato condotto da Rotundo et al. [13], realizzato secondo le regole metodologiche e statistiche indicate dal CONSORT, ha dimostrato che I'uso del laser Er:YAG, come monoterapia o in aggiunta all'approccio meccanico convenzionale, non è efficace (o particolarmente utile, nel caso dell'associazione) se posto a confronto con I'approccio meccanico convenzionale.

Una recente revisione della letteratura [14] ha inoltre evidenziato che anche l'impiego del laser a diodi in terapia causale parodontale non sembra essere efficace.

È necessario altresì ricordare i potenziali effetti collaterali della terapia laserassistita, riportati in numerosi studi.

La laserterapia, a partire degli anni Novanta del secolo scorso, è stata abbondantemente indagata in letteratura: dai dati disponibili è noto che sono stati pubblicati oltre 1.200 articoli originali; di questi, meno di 20 possiedono però le caratteristiche corrette per poter essere inseriti validamente in revisioni sistematiche della letteratura. Nonostante la notevole mole di lavori scientifici pubblicati, che sottintende un vivo interesse della comunità scientifica, non sono ancora apparsi risultati di una minima rilevanza clinica e i costi diretti e indiretti di tali terapie (acquisto delle apparecchiature, maggior tempo clinico per la terapia) devono far riflettere attentamente sul Ioro utilizzo.

Si possono prendere a prestito, per la parodontologia, le conclusioni del comitato scientifico dell'American Dental Association: "Benché i laser abbiano un utilizzo legittimo in odontoiatria, essi non sostituiscono alcuno degli strumenti convenzionali nell'armamentario dell'odontoiatra. Prima di investire nell'acquisto di laser, gli odontoiatri che intendono utilizzarli devono comprendere attentamente le differenze tra i vari tipi, compreso ciò che la letteratura scientifica afferma in proposito" [15].

\section{I COADIUVANTI CHIMICI}

Recentemente è stato introdotto in commercio un nuovo dispositivo medico basato su una soluzione acida solforica sulfurea, sotto forma di gel o liquido, con azione essiccante e disidratante $\left(H y b e n X^{\circledR}\right)$. L'obiettivo è pertanto quello di aggredire non più i batteri bensì la struttura portante del biofilm batterico.

Due serie di casi clinici (in fase di pubblicazione) hanno evidenziato la sua potenziale efficacia del suo impiego sia in campo parodontale per il trattamento non chirurgico di ascessi parodontali, sia in campo implantare per il trattamento non chirurgico delle perimplantiti.

\section{FULL MOUTH DISINFECTION VS TERAPIA A QUADRANTI}

La Full Mouth Disinfection (FMD) nasce come protocollo di studio per evidenziare se la traslocazione batterica da siti non trattati a siti trattati sia o non sia importante da un punto di vista clinico nella guarigione dopo terapia parodontale causale non chirurgica.

Secondo il protocollo originale, il risultato clinico è statisticamente e clinicamente superiore rispetto alla terapia di controllo sia in tasche moderate sia in tasche profonde, con miglioramento via via più marcato al crescere della profondità iniziale.

Viceversa, moltissimi studi che hanno in qualche modo replicato o cercato di replicare queste esperienze non hanno ottenuto i medesimi risultati clinici [15] pur rilevando un'efficacia equivalente della FMD alle tecniche più tradizionali $e$ tuttora considerate lo standard di cura, dimostrando quindi una sostanziale sicurezza anche del protocollo "full mouth". Se si cerca di comprendere il motivo di tali differenze lo si ritrova nei disegni sperimentali. Essi infatti non sono perfettamente identici tra i diversi gruppi di ricerca e in particolare negli studi del gruppo belga di Quyrinen [16]: essendo studi nati e disegnati per massimizzare la differenza fra test e controllo in termini di traslocazione batterica, il gruppo controllo riceve una spaziatura subottimale delle sedute e il gruppo test riceve anche una massiccia dose di antimicrobico locale (clorexidina); negli altri studi tale differenza invece non è al- 
trettanto enfatizzata e il gruppo controllo riceve la terapia tradizionale nel modo standard e spesso il gruppo test non riceve I'aggiunta dell'antimicrobico.

Alcuni di questi lavori però, pur non mostrando differenze significative tra gruppo test e controllo, evidenziano attraverso diversi design sperimentali come il fattore cruciale per ottenere risultati ottimali sia la prevenzione delle ricolonizzazione dei siti già trattati [15].

Il fatto che questo risultato sia raggiunto mediante un trattamento intensivo e rapido, un uso estensivo della clorexidina o uno stringente protocollo di istruzione motivazionale preterapia oltre che con una costante decontaminazione professionale precoce durante tutto il periodo della guarigione sembra esercitare scarsa influenza sulla risposta clinica finale, a patto che una o più di tali modalità venga adottata.

Anche le migliori revisioni sistematiche della letteratura [17] mostrano sostanzialmente un'equivalenza tra le due terapie. Questa equivalenza cela in realtà un vantaggio statisticamente significativo a favore degli approcci full mouth, benché si tratti di differenze di magnitudine talmente piccola da renderle poco importanti da un punto di vista clinico: si sta infatti parlando di un miglioramento inferiore a $0,5 \mathrm{~mm}$ in termini di maggiore riduzione di tasca, di una maggiore riduzione del sanguinamento al sondaggio inferiore al 10\%. Gli autori delle revisioni sistematiche concludono quindi per la sostanziale equivalenza fra le due terapie ed evidenziano come la scelta dell'una o dell'altra debba essere guidata non da una presunta superiorità quanto piuttosto da considerazioni di altro tipo legate alla modalità e logistica della terapia, nonché a valutazioni accessorie come l'invasività della terapia in rapporto alla salute sistemica del paziente o I'opportunità di associare una terapia antibiotica sistemica.

\section{L'ANTIBIOTICO SISTEMICO}

Quando si prescrive una terapia antibiotica necessaria è sempre importante, come raccomandano Silva-Senem et al. nel 2013 [18], tenere presente:

- le proprietà farmacologiche (farmacocinetica e farmacodinamica);

- il dosaggio che raggiunga la concentrazione minima necessaria per essere efficace e che non sia tossico;

- le caratteristiche relative all'ospite, per esempio eventuali problematiche sistemiche che possano interferire con I'assorbimento del farmaco;

- la compliance del paziente, che deve aver capito come as- sumere I'antibiotico e le possibili conseguenze derivanti dal fatto di non eseguire la terapia prescritta correttamente;

- la complessità e diversità del target microbiota (la prescrizione deve essere mirata e specifica contro i batteri patogeni che si vogliono debellare);

- la variazione della flora intestinale;

- I'aumento della suscettibilità ad altre patologie;

- gli eventuali effetti avversi come riportato dal foglietto illustrativo di ogni farmaco;

- il rapporto costo-beneficio, ovvero l'effettiva necessità di prescrivere I'antibiotico sistemico;

- lo sviluppo di resistenze batteriche.

Centrale oggigiorno è - come anticipato - I'ultimo punto, ma utilizzando combinazioni di antibiotici con differenti modalità d'azione, evitando i dosaggi sub-antimicrobici a lungo termine, risparmiando le ultime generazioni di antibiotici e soprattutto evitando I'abuso di prescrizioni è possibile ridurre lo sviluppo di resistenze batteriche.

La terapia sistemica in parodontologia viene utilizzata nei pazienti affetti da parodontite severa o aggressiva, ove il cocktail di van Winkelhoff (amoxicillina e metronidazolo) ha dato risultati statisticamente significativi e risulta oggi il trattamento di elezione per questa patologia quando associato alla terapia non chirurgica con approccio full mouth. La sua efficacia e il suo razionale sono comprovati dall'ampio spettro d'azione e dalla sinergia tra le molecole nonché dal mantenere il tasso nel sangue sopra la concentrazione minima efficace [19]. Questo cocktail è infatti efficace contro la popolazione microbica parodontopatogena, aerobi/anaerobi facoltativi e anaerobi stretti, nella soppressione di Aggregatibacter actinomycetemcomitans e nei benefici che mostra contro Porphyromonas gingivalis.

I risultati che si ottengono non sono solo microbiologici ma soprattutto clinici. Si registrano infatti una riduzione sia del numero di tasche residue, sia delle indicazioni chirurgiche (e di conseguenza anche delle prescrizioni antibiotiche), sia della progressione della patologia da 6 mesi a 2 anni e un'aumentata stabilità parodontale a lungo termine.

Nonostante I'efficacia di questa terapia sia comprovata, va considerata caso per caso e non giustifica l'utilizzo indiscriminato di antibiotici per la risoluzione delle infezioni parodontali [20].

Le indicazioni sono comunque limitate alle parodontiti aggressive generalizzate, alle croniche severe generalizzate, alle 
forme rapidamente progressive in presenza di tasche diffuse e profonde con elevate percentuali di sanguinamento al sondaggio e presenza di essudato purulento.

L'antibiotico sistemico, come emerge dalla rassegna sopra descritta, viene però prescritto anche in fase acuta in presenza di ascessi parodontali o perimplantari. L'antibiotico più prescritto è amoxicillina + acido clavulanico, che tuttavia ha uno spettro d'azione poco idoneo per le infezioni parodontali. In caso di necessità legata alle condizioni sistemiche del paziente (deficit immunitari diretti o indiretti, rischio di endocardite infettiva, immunodepressione) sarebbe più indicato in queste condizioni patologiche somministrare una tetraciclina o I'associazione amoxicillina + metronidazolo oppure evitare la somministrazione sistemica a favore di un farmaco topico riducendo così effetti collaterali e resistenze batteriche.

\section{GLI ANTIBIOTICI TOPICI}

$L^{\prime}$ impiego di antibiotici applicati localmente in siti/tasche parodontali e/o perimplantari rappresenta un'ulteriore strategia terapeutica a potenziamento del trattamento meccanico e in alternativa alla terapia antibiotica sistemica laddove si ravvedano limiti e controindicazioni.

\subsection{PRESUPPOSTI}

Da un punto di vista generale il successo della somministrazione locale di antibiotico si basa su una serie di principi:

- si deve utilizzare un farmaco attivo sulla totalità o maggior parte dei microrganismi patogeni;

- il principio attivo deve essere in grado di entrare in contatto con i batteri bersaglio;

- deve raggiungere concentrazioni terapeuticamente efficaci in situ;

- deve mantenerle per un tempo sufficientemente lungo;

- non deve diffondere o essere assorbito a livello sistemico;

- deve offrire maneggevolezza clinica e comfort per il paziente.

Il soddisfacimento di questi requisiti richiede quindi I'individuazione non solo del farmaco più idoneo ma anche del veicolo o carrier o supporto migliore capace di garantire le proprietà farmacocinetiche sopra elencate.

Per quanto riguarda il principio attivo, volendo rimanere nell'ambito degli antibiotici, i farmaci più studiati sono alcuni di quelli già descritti per la somministrazione sistemica ossia le tetracicline (tetraciclina cloridrato o $\mathrm{HCl}$, doxiciclina e monociclina) e il metronidazolo.
- Le prime, ricordiamo, sono antibiotici batteriostatici, attivi su un ampio spettro di batteri (anaerobi, aerobi/anaerobi facoltativi e anaerobi) e possiedono un'altra peculiarità, ossia la capacità di contrastare localmente la produzione di collagenasi, metalloproteine e interleuchine esercitando un'azione antinfiammatoria locale.

- Il metronidazolo, attivo sugli anaerobi, si era dimostrato efficace soprattutto in associazione con I'amoxicillina nella somministrazione sistemica. L'applicazione locale richiede però una formulazione farmaceutica particolare, da cui dipende la possibilità di soddisfare tutti i requisiti ideali sopra menzionati.

\subsection{TETRACICLINE}

I primi tentativi descritti in letteratura relativamente all'applicazione locale di antibiotici risalgono alla metà degli anni Settanta. Sarà lo studio di Goodson et al. (1979) [21] a stabilire la validità del principio e aprire la strada a successivi sviluppi. L'autore aveva caricato tetraciclina $\mathrm{HCl}$ in piccoli tubi da dialisi e da questi, una volta inseriti nelle tasche parodontali, veniva rilasciata per osmosi. Sebbene la "riserva" di farmaco risultasse esaurita nell'arco di 6 ore, gli autori riportavano effetti positivi dal punto di vista sia microbiologico sia clinico.

Questo studio fornì lo spunto a diversi autori a testare altre molecole ma soprattutto a individuare nuovi metodi di rilascio del principio attivo: ciò rappresenta il filo conduttore della ricerca in tale settore fino ai nostri giorni.

L'evoluzione è consistita nella realizzazione di fibre monolitiche cave in etilenvinilacetato caricate con la quantità nota di $12,7 \mathrm{mg}$ di tetraciclina $\mathrm{HCl}$. Ogni fibra misurava $0,5 \mathrm{~mm}$ di diametro e circa $25 \mathrm{~cm}$ di lunghezza. La fibra veniva inserita all'interno della tasca parodontale dove, stabilizzata mediante cianoacrilato, veniva mantenuta per 7-10 giorni e poi rimossa, essendo non riassorbibile.

Una serie di studi aveva permesso di comprendere meglio le potenzialità del sistema. In primo luogo Tonetti et al. (1990) [22] avevano dimostrato che le concentrazioni di farmaco raggiunte nel fluido crevicolare erano molto elevate e mantenute a lungo ( $1.590 \mu \mathrm{g} / \mathrm{mL}$ per 10 giorni) e che nonostante una certa sostantività della tetraciclina tale effetto era da attribuirsi al veicolo, visto che con la sola irrigazione di soluzioni all' $1 \%$ e al $10 \%$ di tetraciclina $\mathrm{HCl}$ le concentrazioni diminuivano in modo esponenziale, con un tempo di dimezzamento di 4,2 e 12,2 ore rispettivamente. 
Tali misurazioni consentivano di concludere che il sistema "fibre di tetraciclina" permetteva un rilascio costante di farmaco con una cinetica di "ordine zero", ossia non decrescente al diminuire della concentrazione del farmaco stesso, configurando il primo esempio di quello che viene definito sistema a rilascio controllato.

Inoltre Ciancio et al. (1992) [23], in biopsie umane di tessuto gengivale prelevato in tasche in cui erano state applicate le fibre, avevano dimostrato la presenza di tetraciclina nell'epitelio sulculare, e talvolta nel connettivo sottostante, a concentrazioni terapeuticamente efficaci mentre Rapley et al. (1992) [24] avevano riscontrato dosaggi trascurabili, inferiori a 0,1 $\mu \mathrm{g} / \mathrm{mL}$, nel siero di pazienti trattati con le suddette fibre.

Per quanto riguarda l'efficacia clinica diversi studi ne provavano il ruolo come coadiuvante nella terapia non chirurgica con riguardo ai parametri clinici e microbiologici, nella stabilizzazione dei risultati clinici (anche nelle forme recidivanti, sebbene altri studi non osservassero alcun vantaggio dall'associazione tra fibre di tetraciclina e la sola terapia meccanica) [25]. In una recente revisione sistematica, Matesanz-Pérez et al. (2013) [26] hanno valutato i risultati di efficacia clinica di diversi antisettici e antibiotici a uso locale nel trattamento della malattia parodontale. Pur con un certo grado di eterogeneità hanno potuto concludere che I'utilizzo di fibre di tetracicline conferiscono un vantaggio significativo in termini di riduzione della profondità di sondaggio, guadagno di attacco e riduzione dei siti con sanguinamento.

Effetti positivi conseguenti all'uso di fibre di tetraciclina sono riportati anche nel trattamento della perimplantite da Mombelli et al. (2001) [27], che hanno riferito cambiamenti significativi in termini di riduzione del sanguinamento al sondaggio nonché dei parametri microbiologici. I risultati si mantenevano stabili nel periodo di osservazione di 12 mesi.

Nonostante una certa efficacia clinica, il limite principale di tale formulazione risiedeva nel fatto che la tecnica di applicazione fosse indaginosa e richiedesse tempo ed esperienza nonché nella necessità di rimuovere le fibre stesse. Tale prodotto commercializzato come Actisite ${ }^{\circledR}$ non è al momento più disponibile sul nostro mercato.

Sempre nell'ambito delle tetracicline, nel tempo sono stati realizzati prodotti che utilizzano veicoli riassorbibili. Un esempio è un preparato a base di minociclina caricata su microsfere di un polimero riassorbibile che viene inserita sotto forma di polvere nella tasca gengivale. La degradazione del veicolo permette il rilascio per un lungo periodo (2l giorni) (Mino- tek $^{\circledR}$, OraPharma Inc., Amsterdam Zuidoost, Paesi Bassi). Per tipologia di farmacocinetica anche questo prodotto rientra nei sistemi a rilascio controllato e come tale necessita di una sola applicazione.

Diversa invece è la farmacocinetica di un prodotto a base di doxiciclina all'8,5\% che utilizza come veicolo un gel a base di poli-DL-lattide + metil-pirrolidone (Atridox ${ }^{\circledR}$, Olmar Inc., Fort Collins, C0, USA). Questo tipo di prodotto rilascia il principio attivo a seguito del proprio dissolvimento. Per tale ragione segue una cinetica di grado 1 in cui la velocità di rilascio è proporzionale alla concentrazione residua di principio attivo. Questi sistemi vanno quindi incontro a un rapido degrado e spesso si rende necessaria una seconda applicazione. Vengono definiti "sistemi a rilascio sostenuto o lento".

Anche per questi prodotti valgono i principi descritti per le fibre, benché i risultati clinici appaiano più discordanti.

Secondo Matesanz-Pérez et al. (2013) [26] i diversi studi utilizzati nella loro metanalisi suggerivano un effetto positivo della monociclina sulla riduzione della profondità di sondaggio ma non sul guadagno di attacco. I dati relativi alla doxiciclina invece sarebbero positivi sia per il sondaggio sia per il livello di attacco.

\subsection{METRONIDAZOLO}

Vivo interesse ha suscitato sin dai primi anni Novanta il metronidazolo, la cui efficacia sui microrganismi anaerobi era nota e che venne preparato sotto forma di gel biodegradabile. Il gel è composto da una sospensione semisolida di metronidazolo benzoato con un monogliceride (gliceril mono-oleato) e un trigliceride (olio di sesamo) (Elyzol ${ }^{\circledR}$, Gaba Vebas Srl, Roma). Questo prodotto fluido alla temperatura corporea inizia a cambiare stato cristallizzando in contatto con I'acqua del fluido crevicolare. Il cambiamento di stato si associa ad aumento di viscosità e consistenza tanto da trattenere il prodotto nella tasca stessa. Contestualmente iniziano I'idrolisi del metronidazolo e il suo rilascio con un meccanismo a matrice e un lento rilascio.

Secondo Stoltze (1992) [28] dopo 4 ore dalla somministrazione topica di una quantità nota di gel di metronidazolo (pari a $55 \mathrm{mg}$ di principio attivo) la concentrazione nel plasma e nel fluido crevicolare erano pari, rispettivamente, a 0,6 $\mathrm{mg} /$ $\mathrm{mL}$ e $461 \mu \mathrm{g} / \mathrm{mL}$. Questo dato confermava la possibilità di raggiungere concentrazioni locali molto elevate a fronte di un assorbimento sistemico assai modesto.

I benefici clinici di questo prodotto, sia quando confrontato 
rispetto al trattamento non chirurgico da solo o in aggiunta, non sono mai stati dimostrati chiaramente.

In accordo con Matesanz-Pérez et al. (2013) [26] quando si valutano i benefici dell'associazione di antibiotici locali con il trattamento non chirurgico si deve tener conto anche della rilevanza clinica dei dati. Secondo gli autori mancherebbero chiare indicazioni per raccomandarne I'uso nelle tasche profonde e ricorrenti della parodontite cronica. Quando indicati, si raccomanda la scelta di prodotti con una cinetica a rilascio controllato. Gli autori enfatizzano infatti l'importanza del carrier per I'efficacia clinica e quindi come criterio importante nella scelta del prodotto.

\subsection{DOXICICLINA}

Gli antibiotici topici possono migliorare i risultati clinici e microbiologici se associati alla terapia non chirurgica, ma le revisioni della letteratura sull'argomento mostrano che il vantaggio in termini di recupero di attacco clinico rispetto al solo SRP si aggira in media intorno a $0,3 \mathrm{~mm}$ (come per gli antibiotici sistemici): un dato che, se statisticamente significativo, non lo è dal punto di vista clinico. Se però si analizzano i risultati stratificandoli per parodontite cronica o aggressiva si comincia a rilevare una differenza importante.

Nelle forme aggressive, infatti, la somministrazione di un antibiotico topico migliora il risultato del solo SRP in termini di recupero di attacco clinico fino a 0,7 $\mathrm{mm}$ e se si osservano i dati di Eickholz et al. del 2002 [29] emerge che l'aggiunta di doxiciclina al 14\% (Ligosan ${ }^{\circledR}$, Heraeus Kulzer GmbH, Hanau, Germania) allo SRP in pazienti con tasche moderate/severe

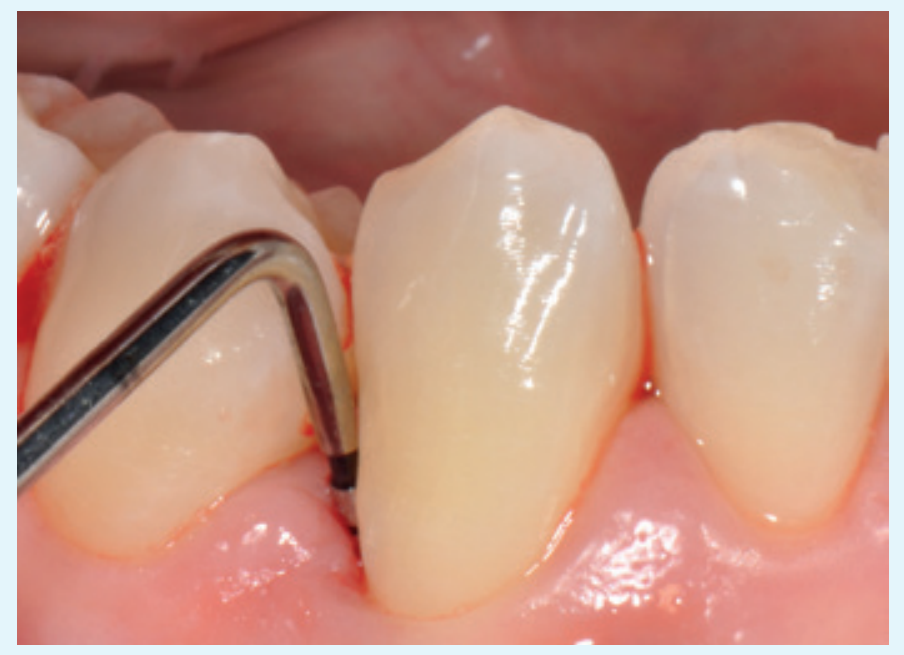

Fig. 1 Caso 1: ascesso parodontale, sondaggio al basale porta a una riduzione media della profondità di sondaggio pari a 3,1 mm, un dato che inizia a diventare rilevante anche dal punto di vista clinico.

Meno significativi i vantaggi in aggiunta allo SRP nei pazienti con tasche residue in terapia di supporto. Sulla scorta della letteratura e dell'esperienza personale, parrebbe che la massima efficacia dell'antibiotico topico $\left(\right.$ Ligosan $^{\circledR}$ ) si ottenga, come con gli antibiotici sistemici, se applicato subito dopo la strumentazione non chirurgica e non sulla tasca residua dopo terapia iniziale [30]. L'altro potente effetto si riscontra se la tasca trattata è in fase attiva con sanguinamento al sondaggio e/o pus (fasi ascessuali o di attività della malattia parodontale). Questo potrebbe spiegarsi considerando che la vascolarizzazione e la vasodilatazione periferica in tali condizioni sono massime e quindi una rapida e importante riduzione della carica microbica subgengivale, grazie all'applicazione dell'antibiotico associata alla successiva azione antinfiammatoria della doxiciclina, permette una guarigione più rapida. Il potenziale rigenerativo delle fibre colliquate durante I'infiammazione acuta infatti è massimo e una rapida eradicazione degli agenti eziologici permette l'espressione di tale capacità.

L'esperienza personale nell'utilizzo di doxiciclina al 14\% nel trattamento degli ascessi parodontali e perimplantari e in associazione alla strumentazione non chirurgica nella terapia delle perimplantiti croniche ha mostrato risultati molto incoraggianti con risoluzione del problema (chiusura delle tasche, scomparsa del sanguinamento e dei sintomi) in tempi brevi. A 48 ore dal drenaggio dell'ascesso attraverso la tasca e dall'applicazione dell'antibiotico si aveva la risolu-

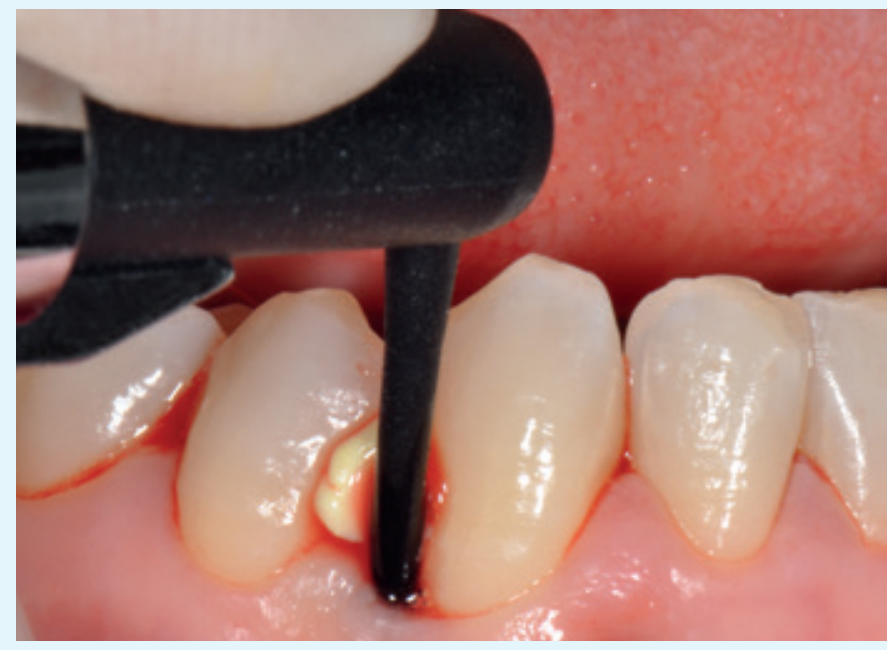

Fig. 2 Caso 1: ascesso parodontale, applicazione di doxiciclina 14\% 


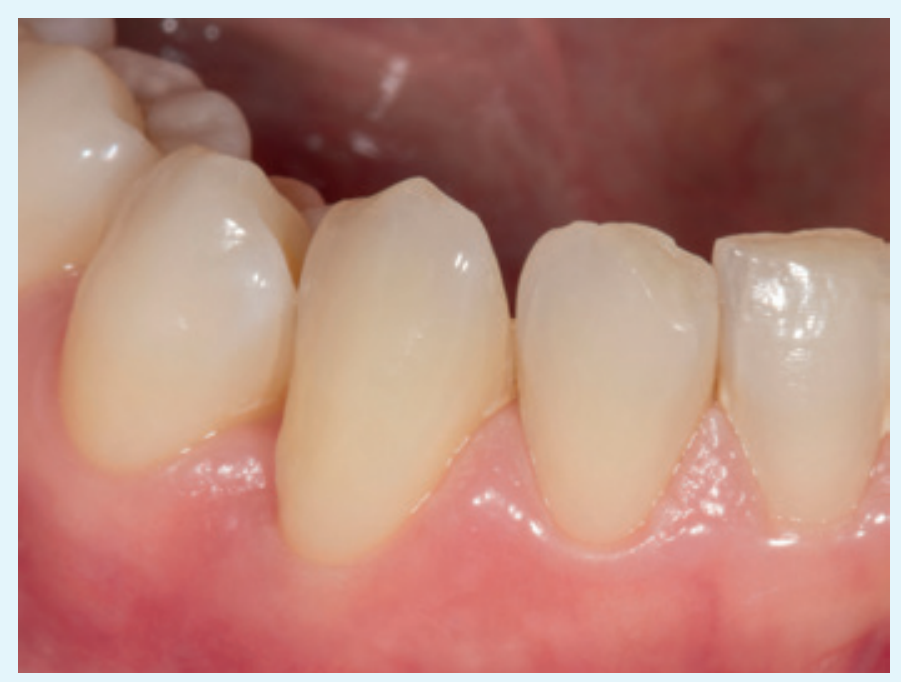

Fig. 3 Caso 1: ascesso parodontale, controllo a 7 giorni

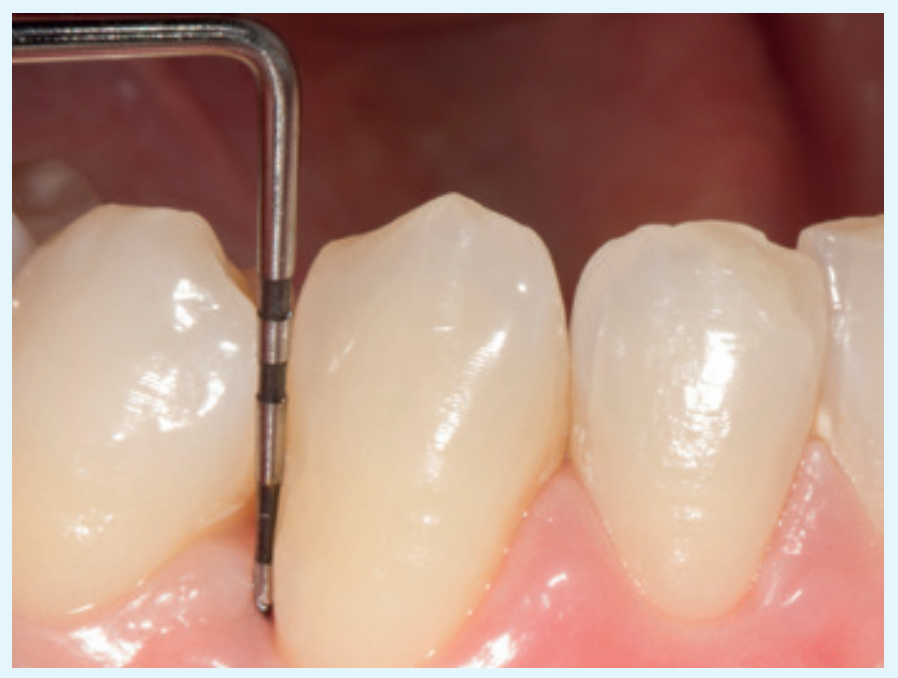

Fig. 4 Caso 1: ascesso parodontale, controllo a 3 mesi

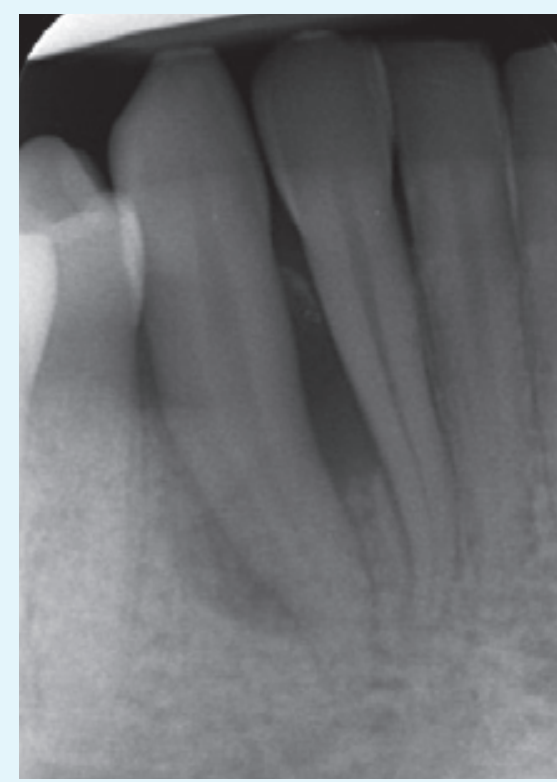

Fig. 5 Caso 1: ascesso parodontale, radiografia al basale

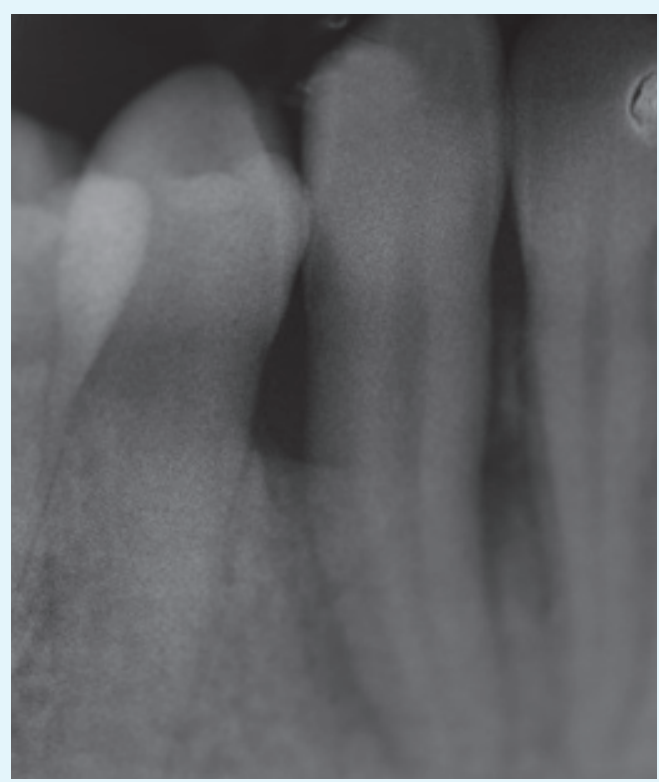

Fig. 6 Caso 1: ascesso parodontale, radiografia di controllo a 3 mesi

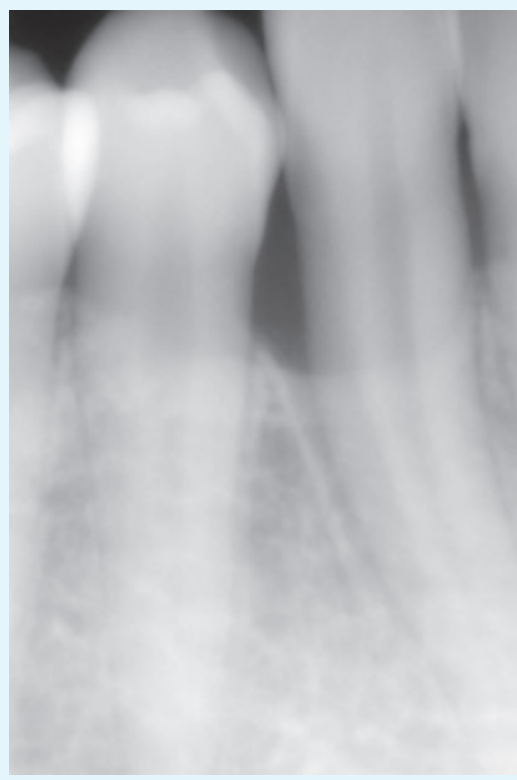

Fig. 7 Caso 1: ascesso parodontale, radiografia di controllo a 1 anno zione completa dei sintomi (dolore, gonfiore, fistola, rossore), dopo una settimana si riscontravano una riduzione della profondità di sondaggio pari al 50\% in media, I'assenza di essudato purulento, la riduzione o scomparsa del sanguinamento al sondaggio e la bonificazione dei tessuti molli. Ciò permetteva di eseguire la terapia non chirurgica adeguata

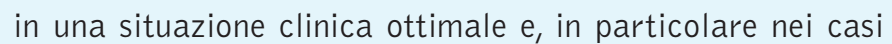
di perimplantite, la seconda applicazione di doxiciclina al
$14 \%$ dopo la terapia non chirurgica garantiva il progressivo miglioramento dei parametri biometrici fino alla chiusura della tasca nella grande maggioranza dei casi a 3 mesi dal trattamento.

I dati di tali studi sono in via di pubblicazione e questi risultati preliminari hanno stimolato a progettare trial clinici randomizzati e controllati a supporto delle prime osservazioni eseguite nelle cases series (figg. 1-12). 


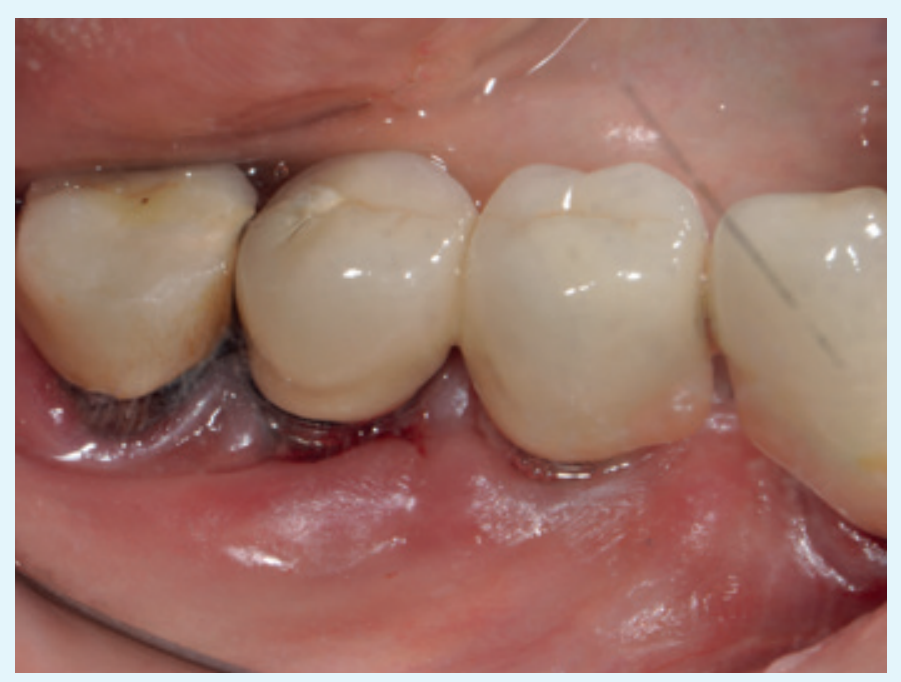

Fig. 8 Caso 2: perimplantite, aspetto clinico al basale

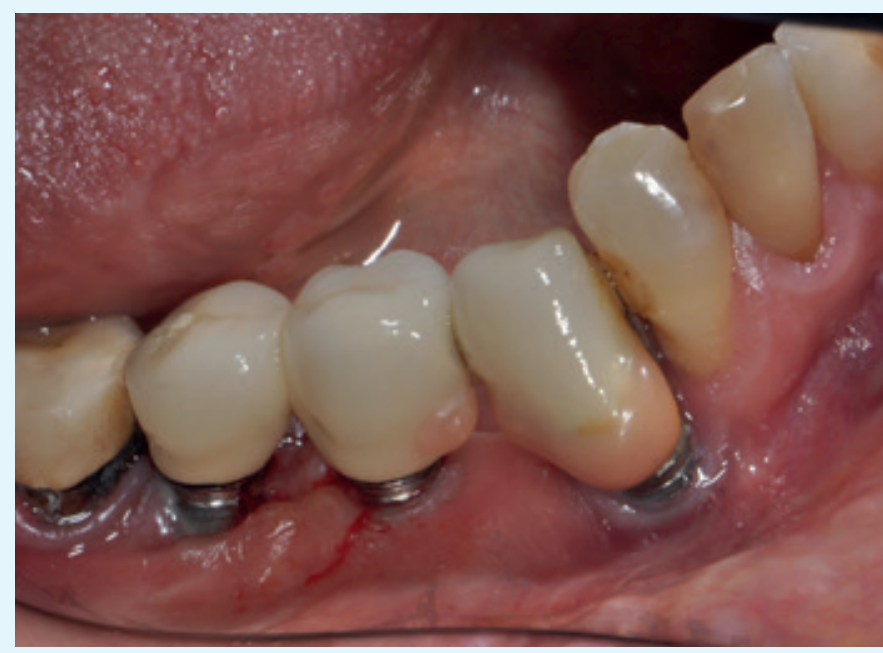

Fig. 10 Caso 2: perimplantite, aspetto clinico a 12 giorni

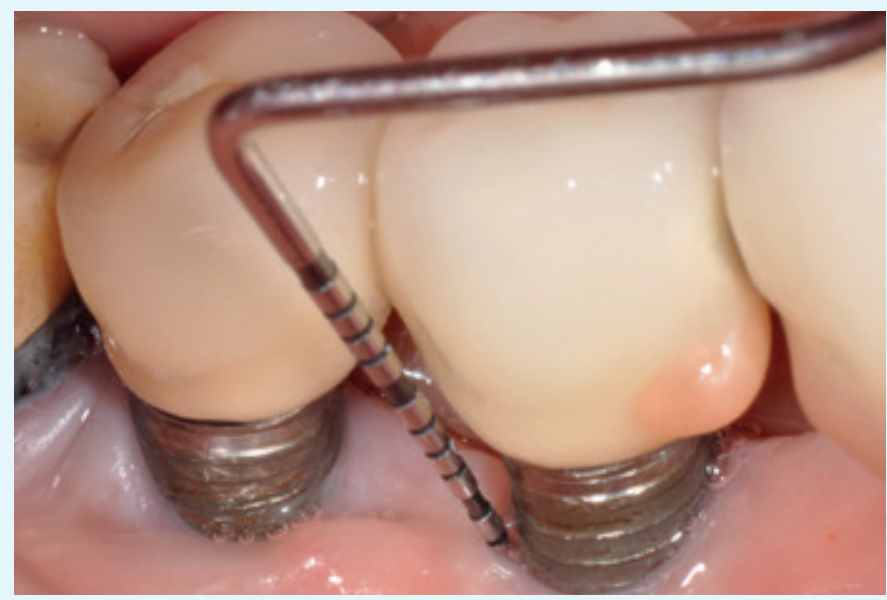

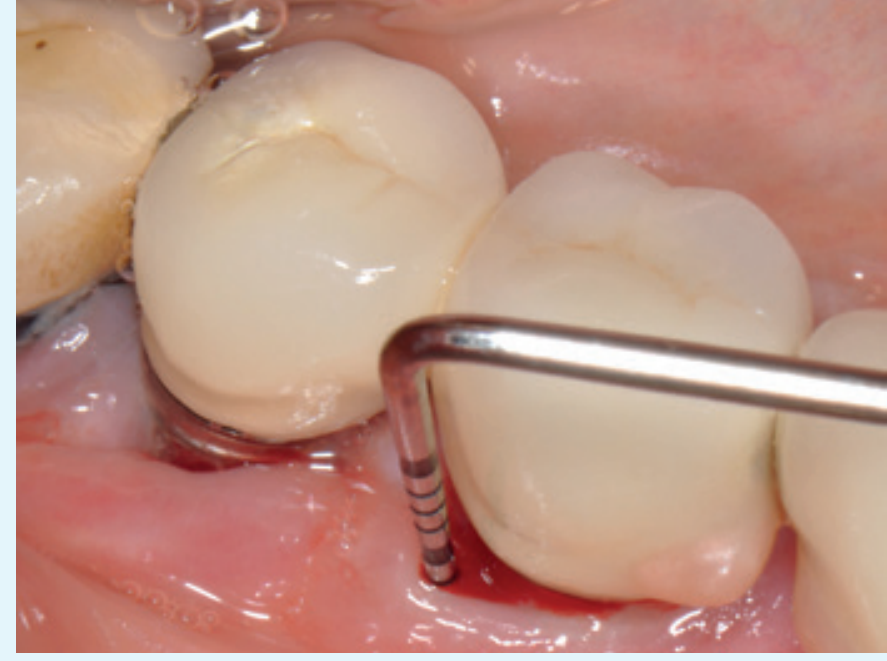

Fig. 9 Caso 2: perimplantite, sondaggio al basale

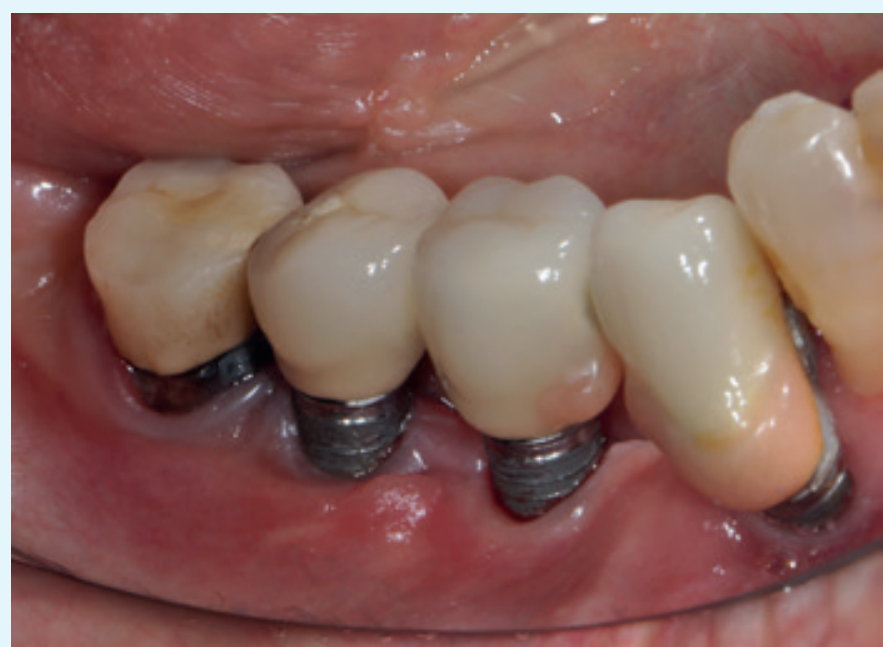

Fig. 11 Caso 2: perimplantite, aspetto clinico a 3 mesi

Fig. 12 Caso 2: perimplantite, aspetto clinico e sondaggio a 1 anno 


\subsection{L'EVOLUZIONE DELLA TECNOLOGIA DEGLI ANTIBIOTICI TOPICI A RILASCIO CONTROLLATO}

Da quanto finora descritto risulta evidente che I'efficacia di un antibiotico topico dipenderà non solo dalla molecola selezionata ma anche dalle caratteristiche del carrier. Le molecole antibiotiche di prima scelta restano le tetracicline, che coprono un ampio spettro batterico compresi gli aerobi/anaerobi facoltativi quali $A$. actinomycetemcomitans, il quale non è eradicato dal metronidazolo, efficace solo verso gli anaerobi stretti (da qui la necessità di associarlo ad amoxicillina).

La concentrazione minima efficace (Minimum Inhibiting Concentration, M IC) del farmaco è un dato la cui conoscenza è di fondamentale importanza. I patogeni parodontali sono sensibili in media a 0,2-1 $\mu \mathrm{g} / \mathrm{mL}$ di tetraciclina $\mathrm{HCl}$. Questo dato viene fornito da studi in vitro i quali però trascurano fattori che influenzano in modo sostanziale la farmacocinetica dell'antibiotico che viene somministrato nell'ambiente parodontale, sottostimandone il reale valore. Il farmaco immesso in una tasca deve contrastare il flusso di fluido crevicolare che tende a diluirlo, deve agire su batteri che sono organizzati e protetti nel biofilm, deve permanere in un ambiente anaerobio, a basso pH ed elevata concentrazione di enzimi, molecole infiammatorie e prodotti batterici. Si comprende quindi la necessità di una somministrazione in dosi molto superiori (anche di 100 volte!) alla MIC indicata da studi in vitro.

È importante altresì sottolineare che il target non è costituito solo dai batteri presenti nel lume della tasca, ma anche da quelli annidati in tessuti duri e molli, quindi cemento e dentina nonché epitelio giunzionale e connettivo subepiteliale. È essenziale che tale elevata concentrazione permanga in situ per il tempo necessario all'esplicarsi dell'azione farmacologica: si ricorda che le tetracicline sono batteriostatiche e quindi I'azione non è così immediata, inoltre devono poter penetrare efficacemente biofilm e tessuti.

Alla luce di tali considerazioni sull'efficacia della molecola e sul rapporto dose/tempo, si è compresa l'esigenza di migliorare il mezzo con cui la molecola stessa veniva somministrata con lo scopo di ottenere caratteristiche ideali di rilascio del farmaco. Il target era un rilascio controllato di concentrazioni molto più elevate della MIC fornita da studi in vitro per più giorni e tramite un carrier possibilmente biodegradabile.

Negli anni sono stati sviluppati carrier con diverse cinetiche di rilascio non solo in odontoiatria, ma in tutti i campi medici con interesse farmacologico. I prodotti sviluppati sono tipicamente distinti come di seguito descritto.

- Rilascio lento o sostenuto (slow-release o sustained-release): sono disponibili sotto forma di complessi (sali o resine), sospensioni, emulsioni e tavolette che rilasciano tutto il farmaco entro 24 ore. Esempio tipico in odontoiatria sono stati i tubi da dialisi in acetato sviluppati da Goodson per il rilascio della tetraciclina $\mathrm{HCl}$, i quali esaurivano la loro riserva di farmaco nell'arco di 4-6 ore. Spesso per queste formulazioni il rilascio è influenzato dall'ambiente in cui vengono inseriti, rendendolo così variabile da paziente a paziente.

- Rilascio controllato (controlled-release): per mezzo di matrici o polimeri con vari meccanismi di diffusione, il farmaco viene rilasciato in concentrazioni predeterminate per un periodo di tempo definito (giorni, settimane, anni). La riassorbibilità è conferita dal fatto che i carrier sono composti da polimeri biodegradabili, per esempio polimeri di acido polilattico-poliglicolico, polivinilpirrolidone, policaprolactone. Man mano che il carrier si biodegrada, il principio attivo viene rilasciato. Esempi di farmaci a uso odontoiatrico sono la doxiciclina $14 \%$ in idrogel biodegradabile, la doxiciclina all' $8,5 \%$ in polimero di polivinilpirrolidone o la tetraciclina in fibre di copolimero etilenvinilacetato. Il rilascio del principio attivo non è influenzato dall'ambiente.

A oggi il mercato italiano offre solo una tetraciclina topica: la doxiciclina al $14 \%$ (Ligosan ${ }^{\circledast}$ ) veicolata da un carrier biodegradabile che ne permette il rilascio controllato [31].

La doxiciclina iclato al $14 \%$ è veicolata da un gel biodegradabile di acido polilattico-poliglicolico, la cui concentrazione arriva a $1.300 \mu \mathrm{g} / \mathrm{mL}$ dopo 2 ore e decresce fino al $70 \mu \mathrm{g} /$ $\mathrm{mL}$ al decimo giorno (fig. 13). È importante sottolineare che la concentrazione raggiunta dipende non dalla percentuale di molecola antibiotica ma dalle caratteristiche del carrier. Nonostante le concentrazioni raggiunte siano ancora inferiori a quelle ottenute con le fibre, sono ampiamente superiori al livello necessario a eradicare i patogeni parodontali. Si tenga conto, per esempio, che per eradicare A. actinomycetemcomitans in vitro bastano $6 \mu \mathrm{g} / \mathrm{mL}$.

Rispetto alle fibre, ha il vantaggio di essere riassorbibile in quanto composta da polimeri biodegradabili, non necessita di impacco parodontale per restare in situ e causa una minore risposta infiammatoria con minore produzione di fluido crevicolare. È da sottolineare anche la capacità di variazione della 


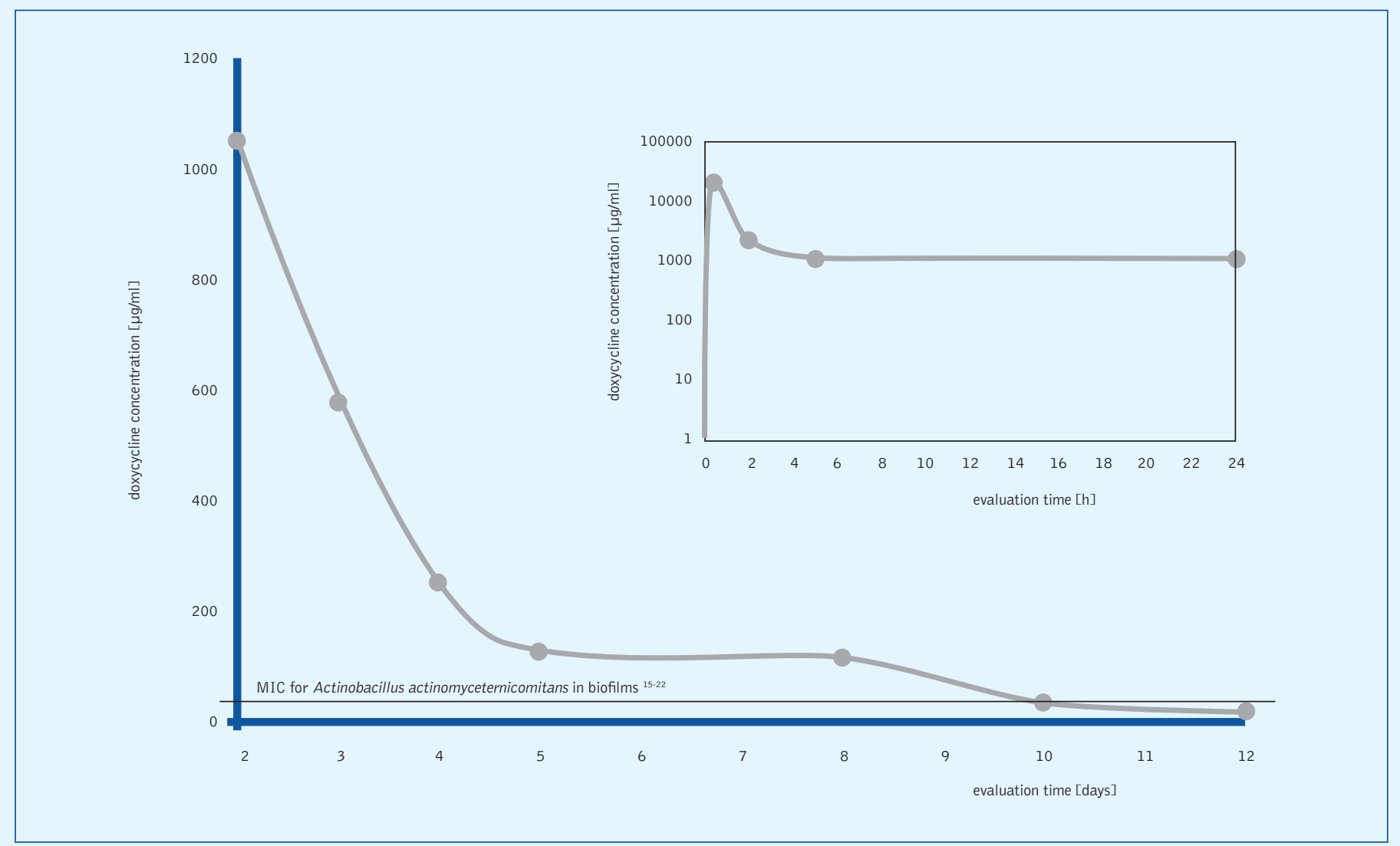

Fig. 13 Cinetica della doxiciclina al $14 \%$ nel fluido crevicolare gengivale

viscosità del prodotto a contatto con I'umidità e il calore. Inizialmente è bassa, in modo da permettere una facile applicazione, per poi aumentare rendendo possibile la permanenza in situ. La consistenza finale si oppone al ritorno elastico dei tessuti dopo l'inserimento e al flusso del fluido crevicolare che normalmente causerebbero I'espulsione del farmaco iniettato nella tasca nel giro di circa 12 minuti.

Vantaggi aggiuntivi sono la monosomministrazione, I'elevata viscosità e il lento riassorbimento del carrier per via idrolitica con il rilascio di prodotti non tossici (acido glicolico, acido lattico) (tab. I).

Le indicazioni al suo utilizzo comprendono siti con infezione acuta, siti con malattia attiva recidivante, siti prechirurgici ancora infiammati e siti affetti da mucosite o perimplantite. L'efficacia clinica è stata ampiamente dimostrata dalla scuola tedesca di Eickholz a partire dal 2002 [29]. Questi autori, tramite un disegno di studio split-mouth, mostrano che applicando la doxiciclina in concomitanza alla terapia meccanica in tasche moderate/severe di pazienti con parodontite grave non trattata o recidivante si ottengono a 3 mesi risultati clinici superiori rispetto a quelli ottenuti con il solo SRP, con diminuzione media della profondità di sondaggio e guadagno di attacco clinico rispettivamente di 3,1 $\pm 1,2 \mathrm{~mm}$ e $2 \pm 1,7 \mathrm{~mm}$

\begin{tabular}{|c|c|c|c|}
\hline \multirow[t]{2}{*}{ onfronto } & \multirow[b]{2}{*}{$\begin{array}{l}\text { Doxiciclina } \\
\text { iclato } 14 \%\end{array}$} & \multirow[b]{2}{*}{\begin{tabular}{|l} 
Doxiciclina \\
iclato $8,5 \%$
\end{tabular}} & \multirow[b]{2}{*}{$\begin{array}{l}\text { Fibre di } \\
\text { tetraciclina }\end{array}$} \\
\hline & & & \\
\hline Carrier & $\begin{array}{l}\text { Macrogol-DL- } \\
\text { Iatttide/glicolide } \\
\text { Poliglicolide }\end{array}$ & \begin{tabular}{|l} 
Poli-DL-lattide \\
N-metil-2- \\
pirollidone
\end{tabular} & Etilenvinilacetato \\
\hline $\begin{array}{l}\text { Concentrazione in situ } \\
\text { al tempo To }(\mu \mathrm{g} / \mathrm{mL})\end{array}$ & 1.300 & 1.085 & 1.300 \\
\hline $\begin{array}{l}\text { Concentrazione in situ } \\
\text { a } 10 \text { giorni }(\mu \mathrm{g} / \mathrm{mL})\end{array}$ & 70 & 46 & 1.300 \\
\hline Biodegradabilità & Sì & Sì & No \\
\hline Viscosità & $>$ & $>>$ & I \\
\hline Risposta infiammatoria & + & + & +++ \\
\hline Applicazione & Singola & Multipla & $\begin{array}{l}\text { Singola }+ \\
\text { rimozione }\end{array}$ \\
\hline \multicolumn{4}{|l|}{ Legenda:/ inserite come solido. } \\
\hline
\end{tabular}


a 6 mesi. Inoltre l'effetto è prolungato e la superiorità diventa ancora più marcata a 6 mesi dalla terapia. È stata appurata anche la sicurezza del prodotto, con osservazione di rarissimi effetti avversi localizzati (gonfiore, fuoriuscita del gel dalla tasca). L'efficacia non è solo clinica ma anche microbiologica. È stata dimostrata un'importante e duratura riduzione della presenza di A. actinomycetemcomitans, T. forsythia, P. gingivalis e T. denticola sia a 3 sia a 6 mesi. È interessante il fatto che il solo veicolo usato come controllo-placebo abbia dato luogo a piccoli miglioramenti a 3 mesi dalla terapia.

Analizzando i casi clinici portati dagli esperti si evince che:

- il farmaco risulta efficace nella risoluzione dei segni e sintomi della fase acuta di ascessi parodontali e perimplantari;

- il farmaco associato a uno specifico protocollo di debridment meccanico perimplantare permette di ottenere dati stabili a un anno (dati in fase di pubblicazione dal parte gruppo della dottoressa Mensi dell'Università degli Studi di Brescia);

- il farmaco associato a debridment nella terapia di supporto in tasche residue riduce in modo non clinicamente significativo la profondità di sondaggio;

- il farmaco nelle tasche residue attive risulta essere efficace;

- il farmaco risulta efficace nella preparazione di un sito chirurgico da rigenerare;

- risulta di facile e veloce applicazione (considerando la forza da esercitare per l'estrusione di un gel viscoso);

- il paziente non in anestesia locale avverte una sensazione di pressione nel sito che può essere fastidiosa;

- non sono stati registrati effetti collaterali;

- il calibro del puntale può risultare di difficile inserimento in siti non infiammati data la non lassità dei tessuti.

Gli esperti sono concordi nell'affermare la necessità di un presidio antimicrobico topico efficace nella risoluzione della patologia perimplantare e parodontale, soprattutto nella fase acuta, evitando così di prescrivere un antibiotico sistemico la cui concentrazione in situ arriva a essere blandamente efficace.

Dal confronto tra gli esperti emerge inoltre che la terapia non chirurgica presenta dei limiti e la disponibilità di un antibiotico topico che migliori in modo clinicamente significativo i risultati di questa terapia sarebbe auspicabile. La doxiciclina iclato al 14\%, che grazie al suo carrier mantiene la MIC efficace per 12 giorni attraverso un rilascio lento e controllato, ha prodotto risultati positivi ed entusiasmanti, soprattutto in ambito perimplantare, e risulta quindi un valido coadiuvante. A fronte di tutto ciò e date le premesse, gli esperti ritengono necessario un trial clinico randomizzato per verificare I'efficacia dell'antibiotico topico soprattutto in ambito perimplantare, dato I'aumento dell'incidenza e della prevalenza della patologia e della sua rilevanza socioeconomica. Sarebbe altresì interessante approfondire la rilevanza dell'utilizzo dell'antibiotico topico nelle tasche residue comparandolo agli altri presidi coadiuvanti.

\section{BIBLIOGRAFIA}

1. Armitage GC. Periodontal diagnoses and classification of periodontal diseases. Periodontol 2000 2004;34:9-21.

2. Guarnelli ME, Zangari F, Manfrini R, Scapoli C, Trombelli L. Evaluation of additional amine fluoride/stannous fluoride-containing mouthrinse during supportive therapy in patients with generalized aggressive periodontitis. A randomized, crossover, double-blind, controlled trial. J Clin Periodontol 2004;31(9):742-8.

3. Guarnelli ME, Farina R, Franceschetti G, Minenna L, Trombelli L. Strumentazione meccanica e antimicrobici domiciliari: effetti microbiologici. Dental Cadmos 2009;77(3):21-34.

4. Hallmon WW, Rees TD. Local anti-infective therapy: mechanical and physical approaches. A systematic review. Ann Periodontol 2003;8(1):99-114.

5. Chapple IL, Walmsley AD, Saxby MS, Moscrop H. Effect of subgingival irrigation with chlorhexidine during ultrasonic scaling. J Periodontol 1992;63(10):812-6.

6. Guarnelli ME, Franceschetti G, Manfrini R, Trombelli L. Adjunctive effect of chlorhexidine in ultrasonic instrumentation of aggressive periodontitis patients: a pilot study. J Clin Periodontol 2008;35(4):333-41.

7. Lang N, Feres M, Corbet E, Ding Y, Emingil G, Faveri M, et al. Group B. Consensus paper. Non-surgical periodontal therapy: mechanical debridement, antimicrobial agents and other modalities. J Int Acad Periodontol 2015;17(Suppl 1):34-6.

8. Sculean A, Aoki A, Romanos G, Schwarz F, Miron RJ, Cosgarea R. Is photodynamic therapy an effective treatment for periodontal and peri-implant infections? Dent Clin North Am 2015;59(4):831-58.

9. Research, Science and Therapy Committee of the American Academy of Periodontology. Lasers in periodontics. J Periodontol 2002;73(10):1231-9.

10. Cobb CM. Lasers in periodontics: a review of the literature. J Periodontol 2006;77(4):545-64.

11. Schwarz F, Aoki A, Becker J, Sculean A. Laser application in non-surgical periodontal therapy: a systematic review. J Clin Periodontol 2008;35(Suppl 8):29-44.

12. Zhao Y, Yin Y, Tao L, Nie P, Tang Y, Zhu M. Er:YAG laser versus scaling and root planing as alternative or adjuvant for chronic periodontitis treatment: a systematic review. J Clin Periodontol 2014;41(11):1069-79.

13. Rotundo R, Nieri M, Cairo F, Franceschi D, Mervelt J, Bonaccini D, et al. Lack of adjunctive benefit of Er:YAG laser in non-surgical periodontal treatment: a randomized split-mouth clinical trial. J Clin Periodontol 2010;37(6):526-33.

14. Slot DE, Jorritsma KH, Cobb CM, van der Weijden FA. The effect of the thermal diode laser (wavelength $808-980 \mathrm{~nm}$ ) in non-surgical periodontal therapy: a systematic review and meta-analysis. J Clin Periodontol 2014;41(7):681-92.

15. Feres M, Gursky LC, Faveri M, Tsuzuki C0, Figueiredo LC. Clini- 
cal and microbiological benefits of strict supragingival plaque contro as part of the active phase of periodontal therapy. J Clin Periodontol 2009;36(10):857-67.

16. Quirynen M, De Soete M, Boschmans G, Pauwels M, Coucke W, Teughels $W$, et al. Benefit of "one-stage full-mouth disinfection" is explained by disinfection and root planing within 24 hours: a randomized controlled trial. J Clin Periodontol 2006;33(9):639-47.

17. Lang NP, Tan WC, Krähenmann MA, Zwahlen M. A systematic review of the effects of full-mouth debridement with and without antiseptics in patients with chronic periodontitis. J Clin Periodontol 2008;35(Suppl 8):8-21.

18. Silva-Senem MX, Heller D, Varela VM, Torres MC, Feres-Filho EJ, Colombo AP. Clinical and microbiological effects of systemic antimicrobials combined to an anti-infective mechanical debridement for the management of aggressive periodontitis: a 12-month randomized controlled trial. J Clin Periodontol 2013;40(3):242-51.

19. Guerrero A, Griffiths GS, Nibali L, Suvan J, Moles DR, Laurell L, et al. Adjunctive benefits of systemic amoxicillin and metronidazole in non-surgical treatment of generalized aggressive periodontitis: a randomized placebo-controlled clinical trial. J Clin Periodontol 2005;32(10):1096-107.

20. Mombelli A. Antimicrobial advances in treating periodontal diseases. Front Oral Biol 2012;15:133-48.

21. Goodson JM, Haffajee A, Socransky SS. Periodontal therapy by local delivery of tetracycline. J Clin Periodontol 1979;6(2):83-92.

22. Tonetti M, Cugini MA, Goodson JM. Zero-order delivery with periodontal placement of tetracycline-loaded ethylene vinyl acetate fibers. J Periodontal Res 1990;25(4):243-9.

23. Ciancio SG, Cobb CM, Leung M. Tissue concentration and localization of tetracycline following site-specific tetracycline fiber therapy. J Periodontol 1992;63(10):849-53.
24. Rapley JW, Cobb CM, Killoy WJ, Williams DR. Serum levels of tetracycline during treatment with tetracycline-containing fibers. J Periodontol 1992;63(10):817-20.

25. Michalowicz BS, Pihlstrom BL, Drisko CL, Cobb CM, Killoy WJ, Caton JG, et al. Evaluation of periodontal treatments using controlled-release tetracycline fibers: maintenance response. J Periodontol 1995;66(8):708-15.

26. Matesanz-Pérez $P$, Garcıa-Gargallo $M$, Figuero $E$, Bascones-Martınez A, Sanz M, Herrera D. A systematic review on the effects of local antimicrobials as adjuncts to subgingival debridement, compared with subgingival debridement alone, in the treatment of chronic periodontitis. $J$ Clin Periodontol 2013;40(3):227-41.

27. Mombelli A, Feloutzis A, Brägger U, Lang NP. Treatment of peri-implantitis by local delivery of tetracycline. Clinical, microbiological and radiological results. Clin Oral Implants Res 2001;12(4):287-94.

28. Stoltze K. Concentration of metronidazole in periodontal pockets af ter application of a metronidazole $25 \%$ dental gel. J Clin Periodontol 1992;19(9 Pt 2):698-701.

29. Eickholz P, Kim TS, Bürklin T, Schacher B, Renggli HH, Schaecken $M T$, et al. Non-surgical periodontal therapy with adjunctive topical doxycycline: a double-blind randomized controlled multicenter study. J Clin Periodontol 2002;29(2):108-17.

30. Ratka-Krüger $P$, Schacher B, Bürklin $T$, Böddinghaus $B$, Holle R, Renggli $\mathrm{HH}$, et al. Non-surgical periodontal therapy with adjunctive topical doxycycline: a double-masked, randomized, controlled multicenter study. II. Microbiological results. J Periodontol 2005;76(1):66-74.

31. Kim TS, Bürklin T, Schacher B, Ratka-Krüger $P$, Schaecken MT Renggli $\mathrm{HH}$, et al. Pharmacokinetic profile of a locally administered doxycycline gel in crevicular fluid, blood, and saliva. J Periodontol 2002;73(11):1285-91. 